\title{
Area Coverage Redundancy and Node Positioning in Wireless Sensor Networks
}

\author{
Priya Kanta \\ Student of DSI
}

\author{
A.M.Prasad \\ Associate professor at DSI
}

\author{
Suma $v$ \\ Dean,RIIC DSI
}

\begin{abstract}
Wireless sensor networks create the platform of an extensive range of applications linked to national security, surveillance, military, health care, and environmental monitoring. One of the most imperative and elementary problems in Wireless Sensor Networks (WSNs) is the coverage problem. Network coverage of wireless sensor network means how well an area of interest is being supervised by the deployed network. The prominence of coverage in WSNs is so important that, it is one of the assets of service parameters. This paper gives information about area coverage redundancy and placing a certain number of nodes such that a region of interest is completely covered and all the nodes and a base station can communicate. This paper considers VDDA(Voronoi diagram deployment algorithm)algorithm for moving distance in the relocation calculation and energy consumption.
\end{abstract}

\section{Keywords}

Wireless Sensor Networks, Coverage redundancy

\section{INTRODUCTION}

Wireless sensor networks [1] are composed of a great number of sensor nodes densely positioned in a manner that may revolutionize information gathering. A sensor node mainly comprises of a sensing unit, a processing unit, a radio transceiver and a power management unit [2]. Sensor nodes produce some measurable responses to the changes in physical or chemical conditions and transmit these responses to a common sink over a wireless channel. The nodes in a wireless sensor network are generally energy-constrained, as the battery of a node may not be recharged. Network coverage is an important issue for WSN. It means how well an area of interest is being monitored by a network. Usually, a node has a limited sensing range. Any event is said to be detectable if at least one node lies within its observable range. A node will cover less area when it is placed near the boundary of the area of interest than when it is placed at the central zone. This is known as boundary or border effect. The reason is that some portion of its sensing area will lie outside the area of interest when it is placed near the border [3]. As pointed out in [4] the coverage concept is measure of the quality of service of the sensing function and is subject to wide range of interpretations due to large variety of sensors and applications. Developing and scaling the WSNs considers the coverage problem and decrease the energy use of the sensors [5].

One of the design optimization strategies is to deterministically place the sensor nodes in order to meet the desired performance goals. In such case, the coverage of the monitored region can be safeguarded through careful planning of node densities and arenas of observation and thus the network topology can be established at setup time. However, in many WSNs applications sensors deployment is random and little control can be employed in order to ensure coverage and yield uniform node density while achieving strongly connected network topology. Therefore, controlled placement is often pursued for only a selected subset of the employed nodes with the goal of structuring the network topology in a way that achieves the desired application requirements. In addition to coverage, the nodes' positions affect numerous network performance metrics such as energy consumption, delay and throughput. For example, large distances between nodes weaken the communication links, lower the throughput and increase energy consumption.

Coverage problem is the most important and basic case in creation of the WSNs. Different classifications are identified for coverage in the sensor networks any of which affect the problem from another point of view.

Area Coverage: The most important problem in area coverage is the coverage kind of an area by the sensors. The main goal of area coverage in WSNs is to cover and supervise environment completely [6]. Any point of under coverage environment in area coverage must be covered at least by one sensor. When an area is covered, any point must be covered by a group of the sensors. So, in this method of coverage we consider a place coordinates and distribute the sensors randomly or manual to cover the aimed environment totally. In area coverage the best coverage takes place when the aimed area is covered by the least number of the sensors completely $[7,8]$. Area coverage is often used for the areas in which the probability of events exists in all coordinates. Also the existence of the redundant sensors in this model cause the multi coverage which lead to the high density of the network.

Point Coverage: The goal is to cover a specific point of the environment in this method. And these points are diffused in the area [9]. It is possible to say that this method is a sub set of the area coverage method. So, if the total area is not covered by the sensors just some points are covered and this means the point coverage. In point coverage just the points which are applicative are covered. So, in point coverage some of the goals are identified by some specific points which must be controlled are considered.

.Barrier Coverage: The total area is not covered by the sensors in barrier coverage. The barrier coverage is a suitable model for penetration diagnosis applications. In this model the coverage operations take place in a way that if the penetration takes place from the width of the under coverage area, the sensors must diagnose them [10]. In barrier coverage the goals are controlled at least by one sensor and all existing goals in that area are under control of that area $[11,12]$.

\section{RELATED WORK}

One of the most important research fields in wireless communications, is WSNs. WSNs includes a set of the sensors which are diffused in supervising environments and process the sensed data and finally send the desired information to the BS(Base Station). Many different methods are presented for improving the coverage problem till now any of which has caused many advances in the coverage and its quality in the WSNs. In the area of adaptive encouragement placement and spatial localization, a number of techniques have been proposed for both fine-grained and coarse-grained localization [13].

The deployment of sensors for coverage of the sensing field has been considered for multi-robot exploration [5]. Each 
robot can be viewed as a sensor node in such systems. An incremental deployment algorithm is used in which sensor nodes are deployed one by one in an adaptive fashion. A drawback of this approach is that it is computationally expensive. As the number of sensors increases, each new deployment results in a relatively large amount of computation

Sensor placement on two- and three-dimensional grids has been formulated as a combinatorial optimization problem, and solved using integer linear programming in [3], [4]. This approach suffers from two main drawbacks. First, computational complexity makes the approach infeasible for large problem instances. Second, the grid coverage approach relies on "perfect" sensor detection, i.e. a sensor is expected to yield a binary yes/no detection outcome in every case. However, because of the inherent uncertainty associated with sensor readings, sensor detection must be modelled probabilistically.

A probabilistic optimization framework for minimizing the number of sensors for a two-dimensional grid has been proposed recently [14].

\section{AREA COVERAGE REDUNDANCY}

Area coverage redundancy is overlapped areas when the agents perform area coverage task, or covered areas that are not in a region of interest. The redundancy does not give productive surveillance. It wastes both time and energy consumption.

The redundancy from overlapped areas usually occurs when an agent are obliged to start and end at the same base station. When an agent completed its area coverage task, it returns to the base station. Its return route repeats the already visited region and causes overlapped area if the final area coverage position is not at the BS.

The overlapped area is determined from the distance between the agent's final area coverage position and the base station. The time spending on area coverage can be minimized by removing an unnecessary path and decreasing the number of turns.

\section{NODE POSITIONING}

Communications gives more robust area coverage algorithm. It allows a base station to know the location and situation of each agent. The base station is able to obtain images from the observed area in near real time using wireless communication system. This is helpful for the base station, especially if the data is critical and time-sensitive. Moreover, it can command the agents to go to specific locations or return to the base station.

Given that the region of interest is in a disaster area or there exists no networking infrastructure, mobile ad hoc networks (MANETs) offer a way to maintain connectivity between the agents. The MANETs communications are performed over radio and do not require any infrastructure. They are very flexible and suitable for several types of applications as they allow the establishment of temporary communication without any pre-installed infrastructure [12].

The node placement can be classified into static positioning and dynamic positioning scheme. In static positioning, the placement of the nodes is determined by the BS before a task begins. The placement calculation is based on the region of interest and sensing range of the nodes.
In dynamic positioning, the placement is determined by each node during the task. The placement calculation for each node is based on current locations of the node and its neighbours, communication range, sensing range and the region of interest. In this dynamic scheme, the nodes find their new locations autonomously and they can reposition themselves when there are one or more missing nodes in the region.

\subsection{Static Positioning of Nodes}

When the environment is sufficiently known, the positions of the nodes can be predetermined. Different clustering shapes could be used to subdivide a region of interest and determine locations of the nodes. The region is subdivided using identical clustering cells and with no overlaps between the cells.

\subsubsection{Minimum Number of Nodes}

The number of agents required to completely cover the region of interest depends on the sensing range and the shape of the region. The distance between the agent and its contiguous neighbours or between the agent and its adjacent boundary must not exceed the sensing range so that the entire region is covered. There is no general solution to find the minimum number of agents required to completely cover the region. The regions with equal area might require different number of agents, if the shapes of the two regions were different.

To uniformly place the nodes in the region, regular hexagonal tiling could be applied. The nodes are placed at the centre of the hexagons. The size of the hexagons corresponds to the node's maximum sensing range to reduce overlapped sensing regions. The hexagons are considered in node positioning if their centres lie inside the region of interest. These hexagons give approximate number and positions of node positioning.

\subsection{Dynamic Positioning of Nodes}

In dynamic positioning, the self-deployment algorithm is considered. The nodes do not rely on the base station for their relocation, but they determine the new locations themselves. The nodes should reach their new locations with minimum time and energy consumption. There are many algorithms for the nodes dynamic repositioning. The nodes are repositioned based on the quality of their performance metrics such as the data rate, sensing range, path length in terms of the number of hops from a sensor node to the base station, etc.

\subsubsection{VD- based Deployment Algorithm (VDDA)}

Voronoi diagram is one of the most fundamental data structures in computational geometry.

Given a number of points in the region of interest, Voronoi diagram can be used to decompose these points into nonoverlapping Voronoi cells (Voronoi region). The cells are divided according to the nearest-neighbor rule. Each given point in the Voronoi cell is associated with the cells of points in the region of interest that are closest to it. These given points and their corresponding Voronoi cells can be applied to the node placement problem by defining the points as the nodes and the Voronoi cells as the communication ranges. Hence, a deployment algorithm for communication can be developed using the notion of Voronoi regions.

This VD-based deployment algorithm (VDDA) is from [14]. The goal of this algorithm is to have the Voronoi cell corresponding to the node's sensing range. The Voronoi cell that matches with a node's sensing range at a certain time instance is considered to be the desired solution in terms of coverage. If the difference between the node's sensing range and the Voronoi cell corresponding to that node are not within 
the predefined tolerance, the node is moved. It is moved until the sensing range and the Voronoi cell are aligned, then the nodal movement is stopped and the resulting solution is accepted.

\section{CONCLUSION}

Dynamic positioning assists the area coverage when the nodes are randomly distributed or when there is a potential node failure. VDDA algorithm use Voronoi diagram to determine the region the node has to cover. VDDA algorithm searches for the location that gives maximal utility gain which is the product of effective area covered by the node at its new location and the estimated lifetime when the node moves to the new location. When applying VDDA to the predetermined node locations with one or more missing nodes, most of the nodes remain at their current locations. This is because the locations are predetermined and the current locations already give maximal utility gain.

\section{REFERENCES}

[1] I.F. Akyildiz, W. Su, Y. Sankarasubrmaniam, E. Cayirci, "A Survey on Sensor Networks", IEEE Communication Magazine, Vol. 40, Issue 8, pp. 102-114, IEEE, Aug 2002.

[2] M. Cardei, D. MacCallum, X. Cheng, M. Min, X. Jia, D. Li, D.Z. Du, "Wireless Sensor Networks with Energy Efficient Organization," Journal of Interconnection Networks, Vol. 3, pp. 213-229, March 2000.

[3] F. Aurenhammer. Voronoi diagrams a survey of a fundamental geometric data structure. ACM Comput. Surv., 23(3):345-405, Sept. 1991.

[4] Y. Bouktir, M. Haddad, and T. Chettibi. Trajectory planning for a quadrotor helicopter. 2008 16th Mediterranean Conference on Control and Automation, pages 1258-1263, 2008.

[5] B. M. Chazelle. Computational geometry and convexity. PhD thesis, New Haven, CT, USA, 1980. AAI8110021.

[6] C. Chen and R.-C. Chang. On the minimality of polygon triangulation. BIT, 30(4):570-582, 1990.
[7] Kh.M. Alam, J. Kamruzzaman, G. Karmakar, M. Murshed, A.K.M. Azad, "QoS Support in Event Detection in WSN through Optimal k-Coverage", Procedia Computer Science, Vol. 4, pp. 499-507, Elsevier, 2011.

[8] J. Yu, X. Deng, D. Yu, G. Wang, X. Gu, "CWSC: Connected K-coverage Working Sets Construction Algorithm in Wireless Sensor Networks", International Journal of Electronics and Communications, Vol. 67, Issue 11, pp. 937-946, Elsevier, 2013.

[9] M. Cardei, M.T. Thai, Y. Li, W. Wu, "Energy-Efficient Target Coverage in Wireless Sensor Networks", 24th Annual Joint Conference of the IEEE Computer and Communications Societies, IEEE, Vol. 3, pp. 1976-1984, 2005.

[10] Chen, S. Kumar, T.H. Lai, "Local Barrier Coverage in Wireless Sensor Networks", IEEE Transactions on Mobile Computing, Vol. 9, Issue 4, pp. 491-504, 2010.

[11] S. Kumar, T.H. Lai, A. Arora, "Barrier Coverage with Wireless Sensors", Proceedings ACM MobiCom, Cologne, Germany, 2005.

[12] B. Liu, O. Dousse, J. Wang, A. Saipulla, "Strong Barrier Coverage of Wireless Sensor Networks",

[13] Proceedings ACM MobiHoc, Hong Kong SAR, China, 2008.

[14] T. Clouqueur, V. Phipatanasuphorn, P. Ramanathan and K. K. Saluja, "Sensor deployment strategy for target detection," In the Proceedings of the 1st ACM international Workshop on Wireless Sensor Networks and Applications (WSNA '02), Atlanta, Georgia, September 2002.

[15] J. Pan, L. Cai, Y. T. Hou, Y. Shi, and S. X. Shen, "Optimal Base-Station Locations in Two-Tiered Wireless Sensor Networks" IEEE Transactions on Mobile Computing, Vol. 4, No. 5, pp. 458 - 473, September 2005. 